\title{
Stem Cells Delivered Oncolytic Virus to Destroy Formidable Brain Tumor
}

\author{
Vignesh Balaji E $\cdot$ K. Sreedhara Ranganath Pai ${ }^{1}$
}

Accepted: 1 November 2021 / Published online: 4 November 2021

(c) The Author(s), under exclusive licence to Springer Science+Business Media, LLC, part of Springer Nature 2021

In brain cancer therapy, various treatment options are available such as surgical resection, radiotherapy, and chemotherapy to fight against malignant cells. But none of the available therapies did not show any promising therapeutic approach to treat cancer patients. Brain cancer is the foremost cause of death worldwide. Nealy, 18 million new cases have been identified every year and causing around 10 million deaths worldwide reported by International Agency for Research on Cancer (IARC) [1]. Most of the patients with advanced brain tumors such as glioblastoma (GBM), neuroblastoma, medulloblastoma, anaplastic astrocytoma have a very minimum survival rate. Also, eradicating glioma-stem cells (GSC) and stimulating the host immune system in the tumor microenvironment are the greatest challenges associated with brain tumor treatment. Cancer cells can effectively hijack the function of the host immune system including tumorinfiltrating lymphocytes (TILs) to supports the development of malignant tissues and cells. Due to the suppression of our body's natural protective agents like immune cells and other specialized cells such as stem cells, tumor cells can home in the infected site and promote their uncontrolled proliferation and growth leads to severe organ failure and death [2].

In that order, activating the host immune response and targeting aberrantly expressed intracellular oncoproteins is an emerging therapeutic approach to attack and destroy the devastating brain tumor. Stem cells (SCs) are considered as a potential immunomodulator that triggers the compromised host immune system by the cancer cells and supports damaged tissue regeneration and repair. Circulating SCs from the multiple tissue origin in the tumor tropism show an ability to home in the area of inflammation and tend to suppress the tumor formation. SCs show an anti-tumor efficacy

\footnotetext{
K. Sreedhara Ranganath Pai

ksr.pai@manipal.edu

1 Department of Pharmacology, Manipal College of Pharmaceutical Sciences, Manipal Academy of Higher Education, Manipal 576104, Karnataka, India
}

via crossing the blood-brain barrier (BBB) and deliver the therapeutic genes to treat the invasive brain tumor It can also be utilized as a carrier for the targeted delivery of the therapeutic molecules to combat tumor cell multiplication and growth [3]. Mesenchymal stem cells (MSCs), also known as mesenchymal stromal cells recognized as adult stem cells originated from various sources mainly found in the bone marrow. It has a capability for self-renewal, multilineage differentiation, injured tissue repair and regeneration, and regulation of the host immune system [3]. Neural stem cells (NSCs), also known as neural precursor cells that can be isolated in the periventricular regions and the subventricular zone of the brain. NSCs tend to migrate themselves to the tumor sites and reduce the tumor mass. Also, it can act for a longer time and inhibits the metastasis potential of the intracranial gliomas via releasing the therapeutic molecules into the targeted site [4]. Hematopoietic stem cells (HSCs) are present widely in the bone marrow employed to form mature blood cells such as red blood cells (RBC), white blood cells (WBC), platelets, and other immune cells like neutrophils, lymphocytes, and macrophages in the body. Induced pluripotent stem cells (iPSCs) are isolated from the undifferentiated inner mass cells of the blastocyst and they can be produced by all types of cells except the placenta cells. iPSCs are the major sources for the host immune cells such as T cells lymphocytes and natural killer (NK) cells and are used as replacement therapy for malignant brain tumor-induced tissue damage and tend to potentiate the host immune system in the tumor microenvironment [5].

Recently, a novel host immune cell booster such as oncolytic virotherapy (OVT) has been introduced in the field of immunotherapy to fight against brain tumors. OVT is an emerging therapeutic approach to target cancer cells and re-activate the suppressed immune system. Genetically engineered oncolytic viruses (OVs) are specifically designed to kill the cancer cells without disturbing the healthy cells and it tends to replicate inside the tumor cells and lyse the tumor mass. Both DNA and RNA viruses 


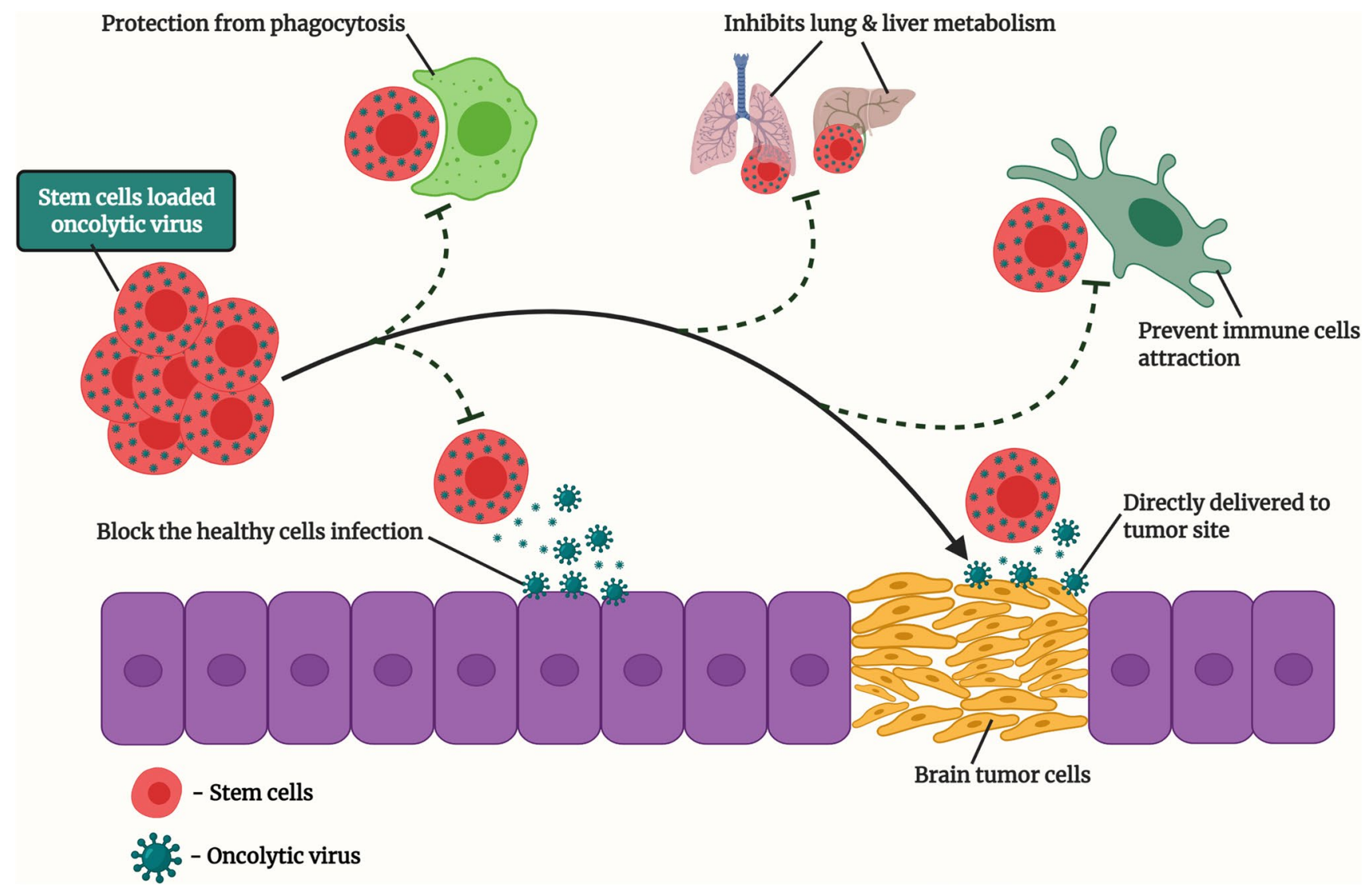

Fig. 1 Optimization of the oncolytic virus using stem cells. Oncolytic viruses (OVs) loaded stem cells (SCs) tend to prevent the biological responses and systemic degradation that enhance the targeted anti-

can kill the tumor cells and enhance the brain cancer patient's survival rate. OVs can stimulate the host's innate and adaptive immune system in the tumor microenvironment to show the protective mechanism against the brain cancer cells [6]. Likewise, OVs can be loaded in various delivery vehicles including SCs to enhance the promising therapeutic efficacy against tumor cells. SCs acts as an emerging delivery vehicle for genetically modified OVs to reach and promote anti-tumor action in tumor cells. SCs is a safe and potential cargo system for OVs that helps to reach the targeted tumor site. SCs act as a protective shield to deliver OVs to safeguard from the liver and lung metabolism. Also, it escapes from the host antiviral immunity and phagocytosis degradation to transport viral particles via the systemic and/or intratumoral route. SCs-carried OVs directly infect the tumor cells without causing any damages to the healthy tissues or cells in the tumor microenvironment (Fig. 1) [7]. Recently, multiple OVs such as adenovirus (AV), herpes simplex virus (HSV), newcastle disease virus (NDV), poliovirus (PV), retrovirus (RV), varicella-zoster virus (VZV), and measles virus (MV) have been tested in the preclinical studies tumor efficacy and significantly debulking the brain tumor cells. Created with BioRender.com

and MSCs and NSCs delivered AV has entered into the clinical trials (NCT03072134 \& NCT03896568) to treat high-grade glioma.

In summary, SCs are a natural cargo system of our body that carries various intracellular and genetic material from one cell to another through paracrine signaling via exosomes and/or on its own. SCs delivered OVs are intended to stimulate the host immune cells such as NK cells, dendritic cells (DC), neutrophils, and TILs to attack the malignant tissues and cells. Nowadays, many harmful human pathogenic viruses such as PV, AV, HSV, RV are genetically modified to use against brain tumor cells. In that order, the current pandemic disease coronavirus (COVID-19) is a single-stranded RNA virus that causes respiratory illness and death worldwide. In the forthcoming days, there is no wonder that SCs loaded genetically engineered COVID-19 might be introduced in the OVT to repress and destroy brain tumor cells. In concluding that SCs delivered OVs might be an excellent therapeutic approach to target the malignant brain cancer with improved safety and efficacy to the patients. 
Acknowledgements All authors thank Manipal College of Pharmaceutical Sciences, MAHE for supporting this work.

Author Contributions VBE and KSRP contributed to the conceptualization of the review article. VB performed an extensive literature search and drafted the manuscript and developed the figures. VB and KSRP reviewed and edited the final manuscript. All authors approved the final draft of the manuscript.

Correspondence to K Sreedhara Ranganath Pai.

Funding No funding was received to assist with the preparation of this manuscript.

Availability of Data and Materials Not application.

\section{Declarations}

Ethical Approval Not applicable.

Consent to Participate Not applicable.

Consent to Publish Not applicable.

Competing Interest The authors declare that they have no competing interests.

\section{References}

1. Dhupal, M., \& Chowdhury, D. (2020). Phytochemical-based nanomedicine for advanced cancer theranostics: Perspectives on clinical trials to clinical use. International Journal of Nanomedicine, 2020(15), 9125-9157. https://doi.org/10.2147/IJN.S259628

2. Rivera-Cruz, C. M., Shearer, J. J., Figueiredo Neto, M., \& Figueiredo, M. L. (2017). The Immunomodulatory Effects of Mesenchymal Stem Cell Polarization within the Tumor Microenvironment Niche. Stem Cells International, 2017, 1-18. https://doi.org/ $10.1155 / 2017 / 4015039$

3. Shah, K. (2016). Stem cell-based therapies for tumors in the brain: Are we there yet? Neuro-Oncology, 18(8), 1066-1078. https://doi. org/10.1093/neuonc/now096

4. Matarredona, E. R., \& Pastor, A. M. (2019). Neural Stem Cells of the Subventricular Zone as the Origin of Human Glioblastoma Stem Cells Therapeutic Implications. Frontiers in Oncology, 9, 1-12. https://doi.org/10.3389/fonc.2019.00779

5. Chu, D. T., Nguyen, T. T., Tien, N. L. B., Tran, D. K., Jeong, J. H., Anh, P. G., Thanh, V. V., Truong, D. T., \& Dinh, T. C. (2020). Recent Progress of Stem Cell Therapy in Cancer Treatment: Molecular Mechanisms and Potential Applications. Cells, 9(563), 1-19. https://doi.org/10.3390/cells9030563

6. Marelli, G., Howells, A., Lemoine, N. R., \& Wang, Y. (2018). Oncolytic viral therapy and the immune system: A double-edged sword against cancer. Frontiers in Immunology, 9, 1-8. https://doi. org/10.3389/fimmu.2018.00866

7. Martinez-Quintanilla, J., Seah, I., Chua, M., \& Shah, K. (2019). Oncolytic viruses: Overcoming translational challenges. The Journal of Clinical Investigation, 129(4), 1407-1418. https://doi. org/10.1172/JCI122287

Publisher's Note Springer Nature remains neutral with regard to jurisdictional claims in published maps and institutional affiliations. 